\title{
Decomposed representation of S- parameters for silicon photonic variation analysis
}

Zhang, Zhengxing, El-Henawy, Sally, Miller, Ryan, Boning, Duane

Zhengxing Zhang, Sally I. El-Henawy, Ryan Miller, Duane S. Boning,

"Decomposed representation of S-parameters for silicon photonic variation analysis," Proc. SPIE 11484, Optical Modeling and Performance Predictions XI, 1148408 (20 August 2020); doi: 10.1117/12.2567731

SPIE. Event: SPIE Optical Engineering + Applications, 2020, Online Only 


\title{
Decomposed Representation of S-Parameters for Silicon Photonic Variation Analysis
}

\author{
Zhengxing Zhang*a, Sally I. El-Henawy ${ }^{\mathrm{a}}$, Ryan Miller ${ }^{\mathrm{b}}$, and Duane S. Boning ${ }^{\mathrm{a}}$

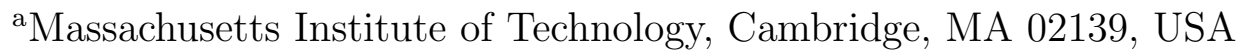 \\ ${ }^{\mathrm{b}}$ Coventor, Inc., 135 Beaver St. Suite 205, Waltham, MA 02452, USA
}

\begin{abstract}
Silicon photonics offers great potential for monolithic integrated photonic and electronic components using existing integrated circuit fabrication infrastructure. However, methods to analyze the impact of IC process variations on performance of photonic components remain limited.

Statistical models based on either simulations or experiments that quantify the effect of these variations are necessary to achieve high-yield manufacturing. In order to cope with the non-linearity in the S-parameters of photonic device components and circuits, non-linear parameter fitting is often used prior to statistical modeling, e.g., rational polynomial fitting of ring resonator responses. The conventional approach treats the amplitude and phase of the S-parameters separately in the fitting process; however, this can be problematic when the behavior of the S-parameters becomes complicated under the variations, since it neglects the strong correlation between amplitude and phase.

We present a novel representation of S-parameters that decomposes the complex-numbered S-parameters into several components each having a simple response that does not require non-linear parameter fitting, and that supports subsequent statistical analysis. We apply the proposed S-parameter decomposition method to Ysplitters with imposed line edge roughness variations. In contrast to the difficulty of the conventional amplitudephase representation, the decomposed representation shows improvement in statistical modeling of variation ensembles, e.g., using principle component analysis.

The method can be extended to other photonic components and circuits with other process variations, to help quantify the effect of process variations for statistical analysis, and to help designers predict and optimize photonic component performance and yield.
\end{abstract}

Keywords: Silicon photonics, process variation, statistical analysis, S-parameters, Y-splitters

\section{INTRODUCTION}

With the increasing demand for higher bandwidth and data rates to meet the requirements of the big data era, silicon photonics rises as an attractive design platform to meet these needs. ${ }^{1,2}$ This is because in silicon photonics light, constituted of non-interacting photons instead of electrons, is used to transmit data. However, except for a few studies, ${ }^{3,4,5,6,7}$ silicon photonics lacks the presence of compact models that fully account for and include the effect of process variation on the behavior of optical components, leading to lower yield and higher cost for photonic integrated circuits. This lack is an important stumbling block that hinders silicon photonics from reaching its full potential.

In optics, S-parameters ${ }^{8}$ are usually used to describe the behavioral change across frequency given various stimuli and inputs. Accordingly, S-parameters are of great importance in the quest to build and develop variationaware compact models that facilitate photonic circuit design and help circuit designers to test the robustness of their design against variations.

In recent work $^{9}$ as part of developing a Y-splitter variation-aware compact model, we consider the effect of process variation by performing a wavelength-wise statistical analysis on the transmission imbalance of Ysplitters ${ }^{10}$ with imposed line edge roughness (LER). To complete the variation-aware compact model of Ysplitters, we now look at other S-parameters. Due to the strong correlation across the wavelengths, the model

*zhxzhang@mit.edu

Optical Modeling and Performance Predictions XI, edited by Mark A. Kahan, Proc. of SPIE Vol. 11484, 1148408 - ( 2020 SPIE - CCC code:

0277-786X/20/\$21 - doi: $10.1117 / 12.2567731$

Proc. of SPIE Vol. 11484 1148408-1 
used in ${ }^{9}$ with only the mean and variance at each wavelength is sufficient to picture the variation of transmission. However, many responses of device components do not have such a simple representation, and instead often exhibit relatively complicated correlation structure across the spectrum.

Typically, principle component analysis $(\mathrm{PCA})^{11}$ is used to transform correlated structures into some number of independent components which are then used to construct simplified or reduced models; this approach implicitly assumes that the variation in the frequency response is small and can be linearized. When using S-parameters to build compact models, the S-parameters are usually described using an amplitude-phase decomposition. In this paper, we show that the traditional amplitude-phase representation of S-parameter can be problematic, causing failure of PCA-based fitting even with relatively small variations. We propose a novel representation and decomposition of S-parameters based on the underlying physics that overcomes the challenges associated with complicated PCA-based fitting, enabling variation-aware compact models.

\section{AMPLITUDE-PHASE REPRESENTATION OF S-PARAMETERS}

S-parameters are used to describe the response behavior, of both electrical and optical devices, as a function of frequency. They are generally complex-valued; hence, in order to perform statistical analysis, we need to work with the full representation of the S-parameters. The traditional approach uses the amplitude-phase representation, where the complex S-parameters are represented by their amplitude $A=|S|=\sqrt{S S^{*}}$ and phase $\phi=\arg (S)=\frac{1}{2 \mathrm{i}} \ln \frac{S}{S^{*}}$, so that

$$
S=A e^{\mathrm{i} \phi}
$$

The amplitude-phase representation has a direct physics interpretation: the amplitude indicates the energy distribution of the device; the phase plays important roles in dealing with interference, and its frequency response is also related to the group delay of the device. Therefore, the amplitude-phase representation is widely used for characterization of device components and compact modeling.

However, this representation is not necessarily optimal for the study of variations. For example, in this paper, we focus on the back reflection of the same Y-splitters with LER as used in the former paper, ${ }^{9}$ where we obtain an ensemble of Y-splitter instantiations by performing virtual fabrication using SEMulator3D, ${ }^{12}$ together with optical simulation using Lumerical FDTD. ${ }^{13}$ As shown in Figure 1, a pattern of ripples exists in the amplitude and group delay* responses of all instantiations, indicating a relatively complicated correlation structure across the spectrum. As the LER amplitude is small in this example, the ripples in most of the instantiations are also small, and thus can be linearized without introducing large error. However, there are some cases with "abnormal" behavior in group delay response (highlighted response in Figure 1b), where there is a sharp spike at certain wavelengths that looks like potential simulation error. But this does not arise due to simulation or numerical error; the trajectory of the S-parameter on the complex plane (highlighted in Figure 1c) does not exhibit any discontinuity, but rather just happens to cross the origin at the wavelength of the spike. This is a characteristic of the amplitude-phase representation: the closer the trajectory moves towards the origin, the faster the phase changes. Therefore, the spike in group delay response must be accompanied by a nearly zero amplitude at the same wavelength. This characteristic becomes a drawback of the amplitude-phase representation for statistical analysis: the amplitude and phase responses of apparently "abnormal" instantiations with trajectory of Sparameters crossing the origin are drastically different from other "normal" responses, which negatively impacts the assumption of small changes needed for linearization. Methods such as PCA attempt to find a continuous Gaussian distribution for all the instantiations, and hence can generate "intermediate" samples (highlighted response in Figure 2b) that make no sense with respect to the physics of the devices, introducing discontinuities in S-parameter trajectory (highlighted in Figure 2c).

\footnotetext{
${ }^{*}$ We use the group delay response as the substitute for the phase response, as it showcases the variation more clearly, while carrying the same amount of information when adding the phase at one frequency point. For the calculation of group delay, please refer to (19).
} 


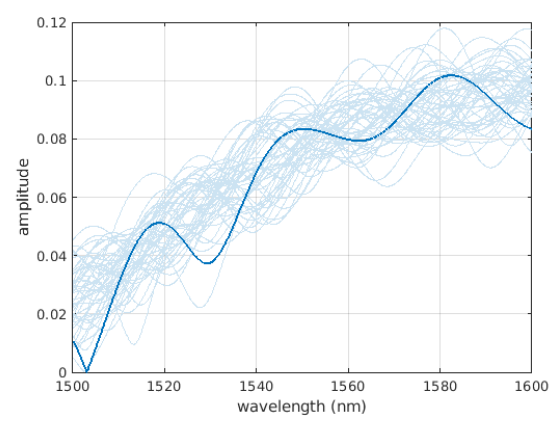

(a) amplitude

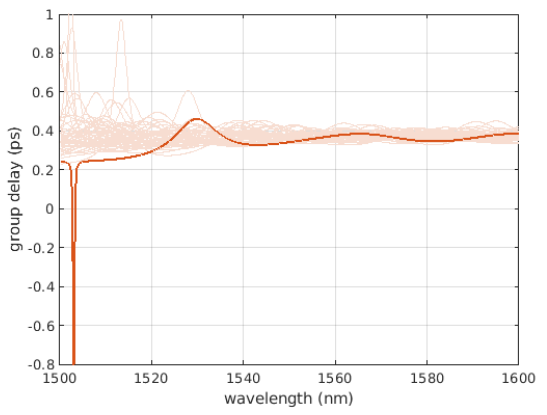

(b) group delay

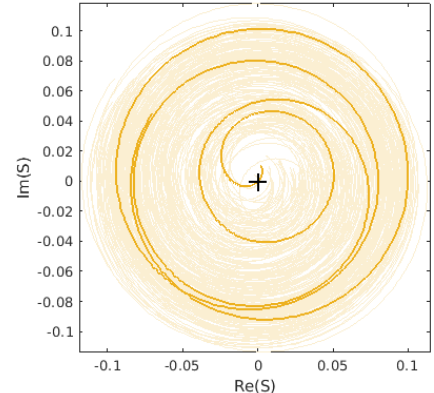

(c) trajectory

Figure 1: Frequency response and S-parameter trajectory of simulated Y-splitter arm back reflection with LER of $A=3 \mathrm{~nm}, L_{c}=40 \mathrm{~nm}$, ensemble of 50 instantiations, with one instantiation highlighted. The black cross marks the location of origin.

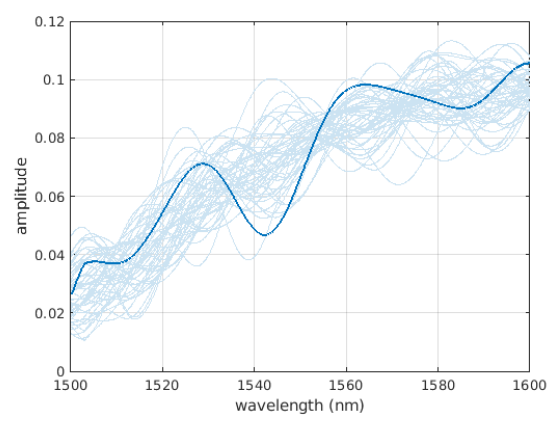

(a) amplitude

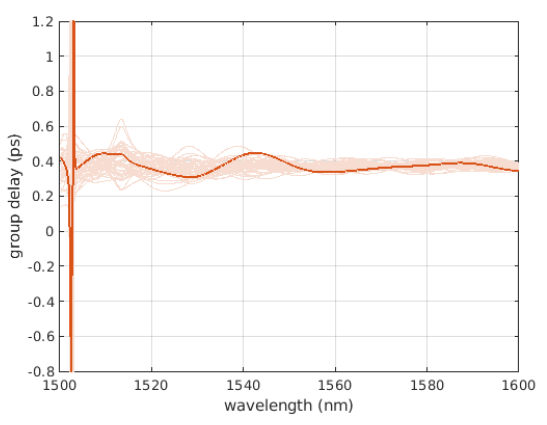

(b) group delay

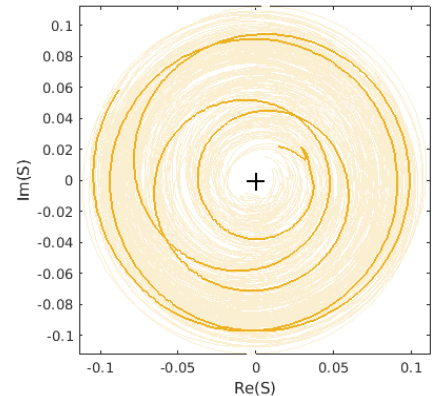

(c) trajectory

Figure 2: Frequency response and S-parameter trajectory of PCA generated Y-splitter arm back reflection with LER of $A=3 \mathrm{~nm}, L_{c}=40 \mathrm{~nm}$ based on the data from Figure 1, 50 instantiations, with one instantiation highlighted. The resulting statistical distribution appears to be different from that shown in Figure 1, and some responses (such as that highlighted) are highly nonphysical with discontinuity.

While the problem seems to be that the PCA and statistical fitting method fails to capture the complicated characteristics and correlation structure in the amplitude and phase responses, it must be understood that the underlying problem is that S-parameters represented in the form of amplitude-phase results in instances that lie far outside the limit of the statistical method. A similar highly nonlinear problem also exists in the responses of resonators, where the problem is solved by modeling some features (e.g., resonant frequency, free spectral range, quality factor, etc. $)^{14}$ upon which the full response can be reconstructed. But in this example, it is uncertain whether one instantiation has spikes (Figure 1b), and how many spikes it has in the frequency range (Figure 3b); thus attempting to extract features from the amplitude or phase response increases the complexity of the problem.

\section{DECOMPOSED REPRESENTATION OF S-PARAMETERS}

To deal with the problem demonstrated by the example in Section 2, one approach is to add a small constant term to the S-parameter so that its trajectory is shifted and away from the origin. However, when the variation becomes slightly larger, the trajectory can come close to the origin at multiple wavelengths (highlighted in Figure 3c), and thus the origin can be "trapped" by the trajectory in a small region. In order to solve the 
problem in a more general and complete manner, we need to start from the basic physics and understand why this phenomenon occurs in silicon photonics.

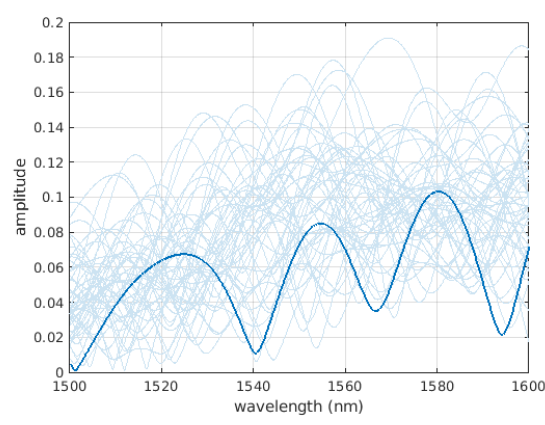

(a) amplitude

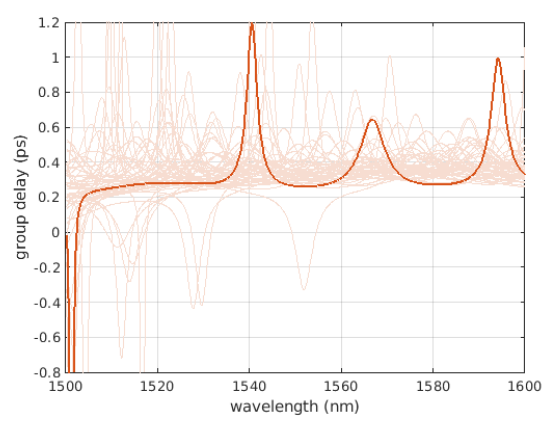

(b) group delay

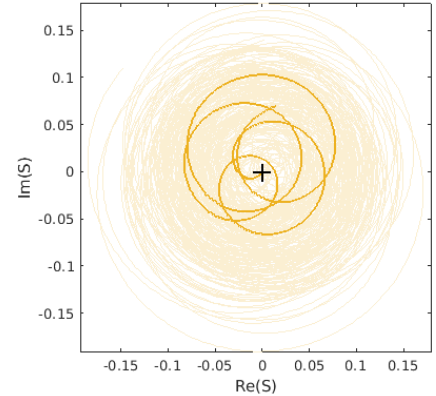

(c) trajectory

Figure 3: Frequency response and S-parameter trajectory of simulated Y-splitter arm back reflection with LER of $A=10 \mathrm{~nm}, L_{c}=40 \mathrm{~nm}$, ensemble of 50 instantiations, with one instantiation highlighted.

Different from electrical devices, optical devices are often subject to and benefit from wave interference. A ring resonator is a good example that makes use of interference to filter out certain frequencies. In our example in Section 2, the back reflection consists of multiple components that are reflected back from different locations of the Y-splitter (Figure 4). At a specific frequency for some particular structure or instance, these components might perfectly cancel each other, which, observed from the complex plane, causes the trajectory of the S-parameter to cross the origin.

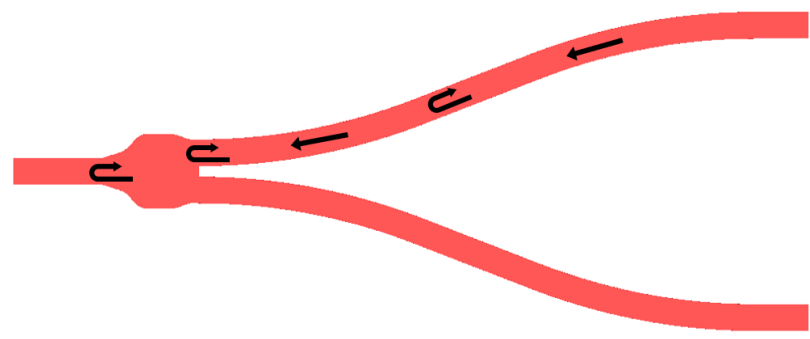

Figure 4: Illustration of the Y-splitter arm back reflection consisting of multiple components that occur at different locations.

In this case, we can formulate the S-parameter in terms of multiple small components:

$$
S=S^{(1)}+S^{(2)}+\cdots+S^{(n)}
$$

where each small component $S^{(i)}$, which we refer to as a "simple" S-parameter, has almost linear amplitude and phase response in the frequency range of interest. In particular, we can divide the simple S-parameters into two types depending on whether its trajectory crosses the origin in the complex plane: Type I simple S-parameters with a spiral-shaped trajectory that does not cross the origin (Figure 5), and Type II simple S-parameters with a heart-shaped trajectory that does cross the origin exactly (Figure 6). While Type I simple S-parameters naturally have well-behaved amplitude and phase response that can be modeled easily, Type II simple S-parameters have an extra $\pi$ jump in phase response at the frequency of origin crossing (solid line in Figure 6b), but which can be eliminated by allowing negative amplitude and flipping the sign of amplitude at one side of the frequency (dashed lines in Figure 6a and Figure 6b). This technique does not apply to all S-parameters in general, because it requires the S-parameter to cross the origin exactly. 


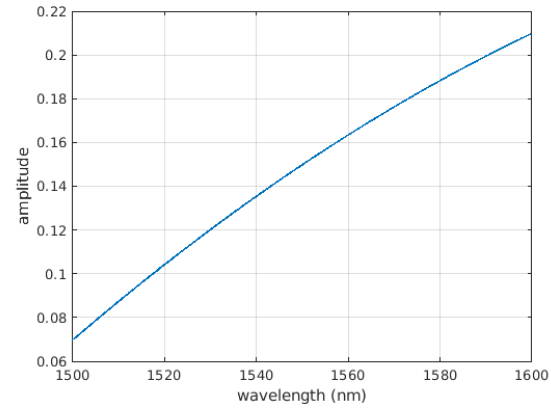

(a) amplitude

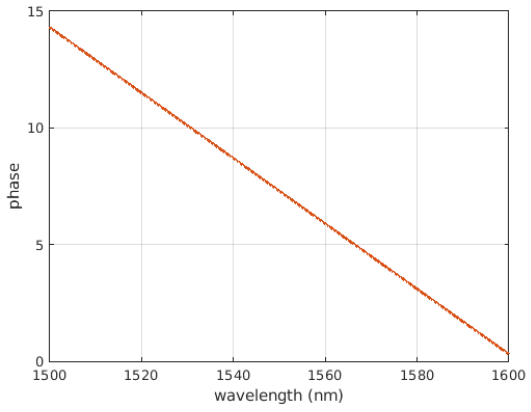

(b) phase

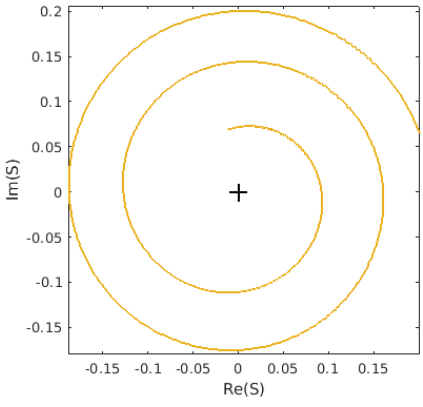

(c) trajectory

Figure 5: Frequency response and S-parameter trajectory of a type I "spiral-shaped" simple S-parameter example.

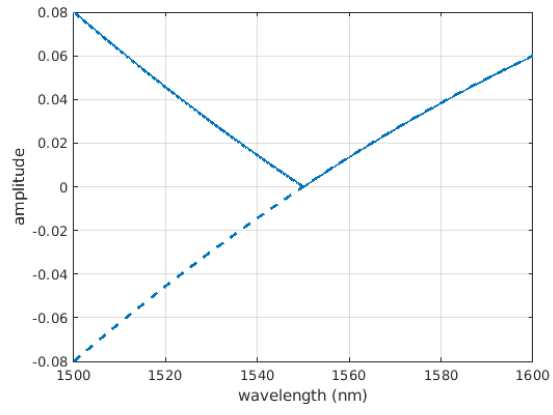

(a) amplitude

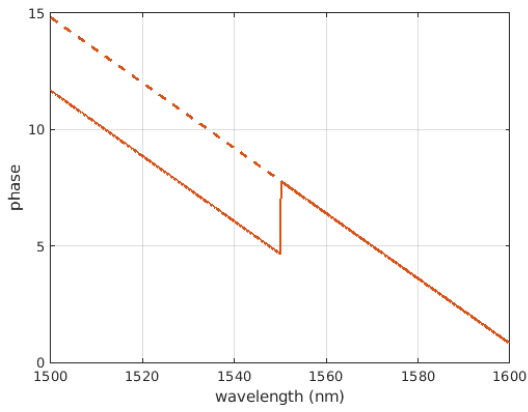

(b) phase

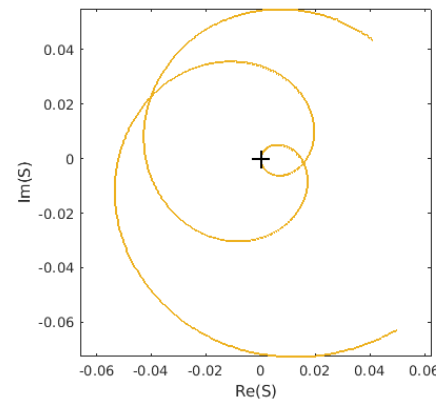

(c) trajectory

Figure 6: Frequency response and S-parameter trajectory of a type II "heart-shaped" simple S-parameter example. Dashed lines show the frequency response after amending the $\pi$ jump crossing origin.

Therefore expressed in amplitude-phase form (with real-valued amplitude and phase), (2) can be written as

$$
S=A^{(1)} e^{\mathrm{i} \phi^{(1)}}+A^{(2)} e^{\mathrm{i} \phi^{(2)}}+\cdots+A^{(n)} e^{\mathrm{i} \phi^{(n)}} .
$$

Due to the relatively straightforward behavior of the simple S-parameters, we expect that statistical analysis of the variation of these components $A^{(1)}, \ldots, A^{(n)}, \phi^{(1)}, \ldots, \phi^{(n)}$ instead of $A$ and $\phi$ in (1) will overcome the nonlinear issue identified in Section 2. As opposed to the single amplitude-phase representation in (1), we call (3) the "decomposed" representation. Since a complex number can be represented fully by two real numbers, the decomposed representation has redundancy and thus has an infinite number of possible solutions (combinations of $A^{(i)}$, or $\phi^{(i)}$ values). Our next goal is to find the decomposition that works best for variation analysis, and is consistent for all instantiations in an ensemble of devices.

\section{DECOMPOSITION: THE FITTING APPROACH}

For the purpose of statistical analysis, we want the decomposed representation components to be as simple as possible. The simplest S-parameters should have polynomial response:

$$
\hat{A}(f ; a)=\sum_{k=0}^{P} a_{k} f^{k}, \quad \hat{\phi}(f ; p)=\sum_{k=0}^{Q} p_{k} f^{k}
$$


where $a=\left(a_{0}, \ldots, a_{P}\right)$ and $p=\left(p_{0}, \ldots, p_{Q}\right)$ are the polynomial coefficients. Hence starting from the simplest S-parameters, we can try to find their combination

$$
\hat{S}(f ; \mathbf{a}, \mathbf{p})=\sum_{i=1}^{n} \hat{S}\left(f ; a^{(i)}, p^{(i)}\right)=\sum_{i=1}^{n} \hat{A}\left(f ; a^{(i)}\right) \exp \mathrm{i} \hat{\phi}\left(f ; p^{(i)}\right)
$$

that is closest to the target $S(f)$. Equivalently, this can be treated as a fitting problem parameterized by the polynomial coefficients $\mathbf{a}=\left(a^{(1)}, \ldots, a^{(n)}\right), \mathbf{p}=\left(p^{(1)}, \ldots, p^{(n)}\right)$. These coefficients operate similar to the features extracted from ring resonator responses, but in our decomposed representation, they are extracted from the complex-numbered fitting.

Although it can be inferred from the physics of the device structure, the number of components $n$ in the decomposed representation (3) still can be hard to determine. One feasible approach is to start from small numbers, e.g., $n=2$, as small decompositions are easy to fit. Then we can determine if more components need to be added depending on the residual of the fitting. In our examples considering the back reflection of Y-splitters, we find that $n=3$ is sufficient for the decomposition.

To avoid bad conditioning and coefficient scaling issues in the fitting, we make a linear transformation on the frequency $f$ :

$$
f \mapsto \frac{2 f-f_{\max }-f_{\min }}{2\left(f_{\max }-f_{\min }\right)}
$$

so that the transformed frequency region of interest becomes $[-1,1]$.

\subsection{First Order Fitting}

We start from the first order case: $P=Q=1$ in (4), and try to find the coefficients through nonlinear least square fitting, i.e., to solve the optimization problem

$$
\left(\mathbf{a}^{*}, \mathbf{p}^{*}\right)=\arg \min _{\mathbf{a}, \mathbf{p}}\|r(f)\|^{2}
$$

that minimizes the sum of square residual

$$
\|r(f)\|^{2}=\sum_{j=1}^{N_{f}}\left|r\left(f_{j}\right)\right|^{2}=\sum_{j=1}^{N_{f}}\left|S\left(f_{j}\right)-\hat{S}\left(f_{j} ; \mathbf{a}, \mathbf{p}\right)\right|^{2}
$$

Minimizing (7) is a nonlinear optimization problem which can be solved using gradient or Newton methods. However, it is hard to find a good initial value due to the large number of parameters. To simplify the problem, we observe that the amplitude terms in (5) can be expanded as

$$
\hat{S}(f ; \mathbf{a}, \mathbf{p})=\sum_{i=1}^{n}\left[\exp \mathrm{i} \phi\left(f ; p^{(i)}\right)\right] a_{0}^{(i)}+\left[f \exp \mathrm{i} \phi\left(f ; p^{(i)}\right)\right] a_{1}^{(i)}
$$

and thus $\hat{S}$ is linear in $\mathbf{a}$. When $\mathbf{p}$ is fixed, the nonlinear least square fitting becomes a linear ordinary least squares (OLS) problem for a, and therefore it can be solved analytically. Letting

$$
L(\mathbf{p})=\min _{\mathbf{a}}\|r(f)\|^{2}
$$

be the optimal value of the OLS with the optimal solution $\mathbf{a}^{*}(\mathbf{p})$, the optimization problem (7) then becomes

$$
\mathbf{p}^{*}=\min _{\mathbf{p}} L(\mathbf{p}), \quad \mathbf{a}^{*}=\mathbf{a}^{*}\left(\mathbf{p}^{*}\right) .
$$

Fitting for $\mathbf{p}$ is still nonlinear, but now with many fewer parameters that need to be initialized: $\hat{S}$ is periodic in $p_{0}^{(i)}$ since it is the shift in phase, thus its initialization is not very sensitive; $p_{1}^{(i)}$ is related to the group delay of the $i$-th component, and thus can be estimated from the geometry of the device structure. 
For the nonlinear fitting problem (11), the solution is sensitive to the initial values of $p_{1}^{(i)}$. Bad initial values can end up trapped in some local minimum of bad solutions, especially when working with a relatively large number of components. However, it is easy to differentiate a bad fitting from a good fitting. Figure 7 shows three different fitting results of the same S-parameter instantiation starting from different initial values. The bad fitting example (Figure 7c) not only results in a significantly larger sum of squared residuals, but also contains two components with similar group delay: $p_{1}^{(1)}=15.7$ and $p_{1}^{(2)}=14.4$. This phenomenon of similar components commonly exists in all of the bad overfitting results and thus can be used as a metric to evaluate the fitting results.
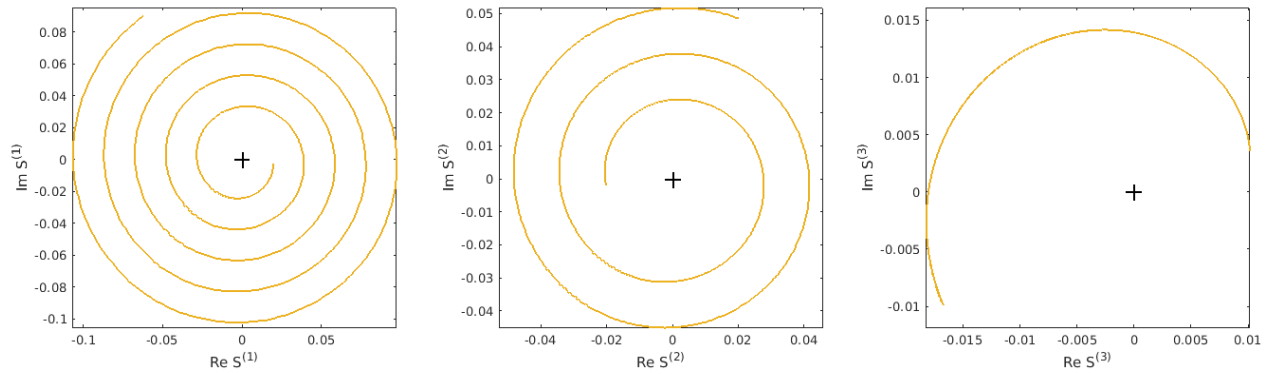

(a) Good fitting: $p_{1}^{(i)}=(14.6,7.3,1.7)$, sum of squared residuals $=0.018$.
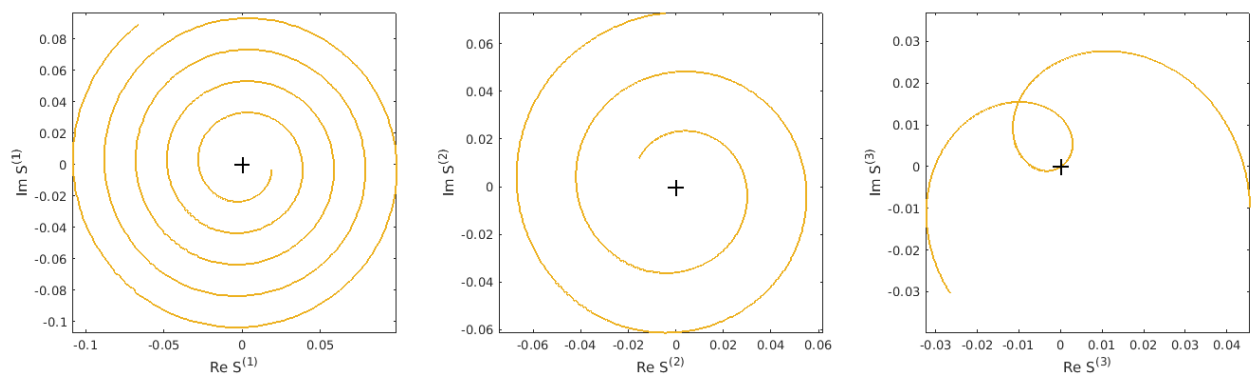

(b) Good fitting: $p_{1}^{(i)}=(14.5,6.7,3.7)$, sum of squared residuals $=0.031$.
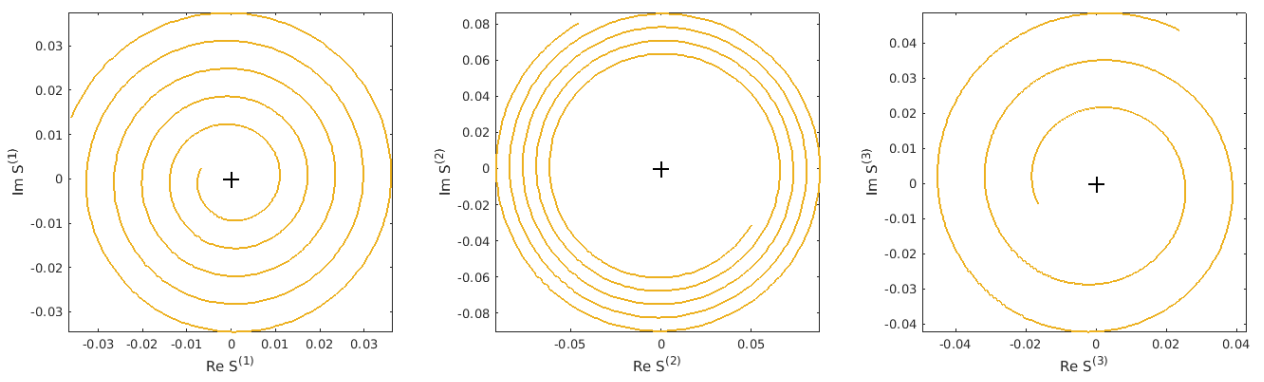

(c) Bad fitting: $p_{1}^{(i)}=(15.7,14.4,7.5)$, sum of squared residuals $=0.205$.

Figure 7: Simple S-parameter components from the first order fitting, same instantiation starting from different initializations.

\subsection{Ensemble: Regularized Fitting}

As shown in Figure 7, there may be different decomposition results starting from different initial values, even though they are all "good" fittings (Figure 7a and Figure 7b). For modeling a single device, one can choose an arbitrary solution if they have similar (or identical) level of residual; but for statistical analysis on an ensemble of devices, it is necessary to keep the decomposition consistent across all of the instantiations. Equivalently, we need to keep the polynomial coefficients $\mathbf{a}, \mathbf{p}$ similar for all of the instantiations. Therefore, we introduce a 
regularization term to the fitting problem (7) when considering an ensemble of instantiations:

$$
\left(\mathbf{a}^{*}, \mathbf{p}^{*}\right)=\arg \min _{\mathbf{a}, \mathbf{p}}\|r(f)\|^{2}+\lambda \sum_{i=1}^{n} \sum_{k=0}^{1}\left(\left|\frac{a_{k}^{(i)}-m_{i k}^{a}}{\sigma_{i k}^{a}}\right|+\left|\frac{p_{k}^{(i)}-m_{i k}^{p}}{\sigma_{i k}^{p}}\right|\right)
$$

where $m_{i k}^{a}$ and $\sigma_{i k}^{a}$ are the ensemble mean and standard deviation of $a_{k}^{(i)} ; m_{i k}^{p}$ and $\sigma_{i k}^{p}$ are the ensemble mean and standard deviation of $p_{k}^{(i)}$. Note that these ensemble parameters do not exist prior to the regularized fitting; however, we can use the results from the first order fitting as an estimate, and then update these parameters during the regularized fitting. After a few iterations of doing regularized fitting from the estimated ensemble parameters and calculating the ensemble parameters from regularized fitting, we might hope that the estimates converge.

However, these iterations never fully converge. The regularized term in (12) tends to shrink the distribution of the polynomial coefficients, and in return, the shrunken distribution provides even smaller ensemble standard deviation for the next iteration, which then further shrinks the distribution. But it should be kept in mind that the purpose of the regularized term is to pick a consistent decomposition when there are multiple solutions, so the penalty parameter $\lambda$ is chosen to be small enough so that it does not change these solutions significantly. With a small amount of penalty, the shrinking issue is not a significant concern during the first few iterations. In addition, the regularized fitting does not require high precision of the ensemble parameters, so a few iterations is sufficient.

Another practical issue in the regularized fitting is to guarantee a globally optimal solution. Similar to the first order fitting in Section 4.1, fitting for a with $\mathbf{p}$ fixed becomes LASSO ${ }^{15}$-like regression and can be solved efficiently without initialization. Optimization for $\mathbf{p}$ can start from the ensemble mean as initial value, but this still does not guarantee convergence to the global minimum. There are several properties about the coefficients of the simple S-parameters that are helpful:

- Period in $p_{0}$ : for the $i$-th component, $p_{0}^{(i)}+2 N \pi$ corresponds to the same S-parameter for any integer $N$;

- $\pi$ jump in $p_{0}$ : for the $i$-th component, $\left(a^{(i)}, p_{0}^{(i)}\right)$ and $\left(-a^{(i)}, p_{0}^{(i)}+\pi\right)$ correspond to the same S-parameter;

- $\pi$ jump in $p_{1}$ : if $\mathbf{p}$ is a local minimum for the fitting (11), then for the $i$-th component, changing $p_{1}^{(i)}$ to $p_{1}^{(i)} \pm \pi$ is likely to fall close to another local minimum.

After one local minimum is found, these properties can be used to search across all of the possible local minima near the ensemble mean, and thus find the global minimum.

\subsection{Higher Order Fitting}

The first order cases may not be sufficient to describe the components in the decomposed representation. In such case, we can include higher order terms in (4) to add some curvature to the simple S-parameters. However, introducing higher order polynomial terms can easily cause overfitting (Figure 8), hence we need to introduce another regularization:

$$
\left(\mathbf{a}^{*}, \mathbf{p}^{*}\right)=\arg \min _{\mathbf{a}, \mathbf{p}}\|r(f)\|^{2}+\lambda_{0} \sum_{i=1}^{n} \sum_{k=0}^{1}\left(\left|\frac{a_{k}^{(i)}-m_{i k}^{a}}{\sigma_{i k}^{a}}\right|+\left|\frac{p_{k}^{(i)}-m_{i k}^{p}}{\sigma_{i k}^{p}}\right|\right)+\lambda_{1} \sum_{i=1}^{n}\left(\sum_{k=2}^{P}\left|\frac{a_{k}^{(i)}}{\hat{a}_{i}}\right|+\sum_{k=2}^{Q}\left|\frac{p_{k}^{(i)}}{\hat{p}_{i}}\right|\right)
$$

where the parameters $\hat{a}_{i}$ and $\hat{p}_{i}$ are chosen to be comparable to the amplitude and phase of the $i$-th component, and the introduced $\lambda_{1}$ penalty can be interpreted as ensuring that the changes introduced by higher order terms should be small compared to the entire trend. In practice, the higher order terms can be determined from the first order fitting. In our example, the first order regularized fitting (12) is performed before introducing higher order terms, and the results are used to initialize the higher order fitting, as well as setting the parameters $\hat{a}_{i}$ and $\hat{p}_{i}$ to:

$$
\hat{a}_{i}=\max _{k=0,1}\left|a_{k}^{*(i)}\right|, \quad \hat{p}_{i}=\pi
$$


from the first order fitting results. Since (13) uses the $\ell_{1}$-norm as the penalty, the higher order terms will not be introduced at all if the first order fitting is sufficient. In our example, only a few instantiations actually introduce the higher order terms.
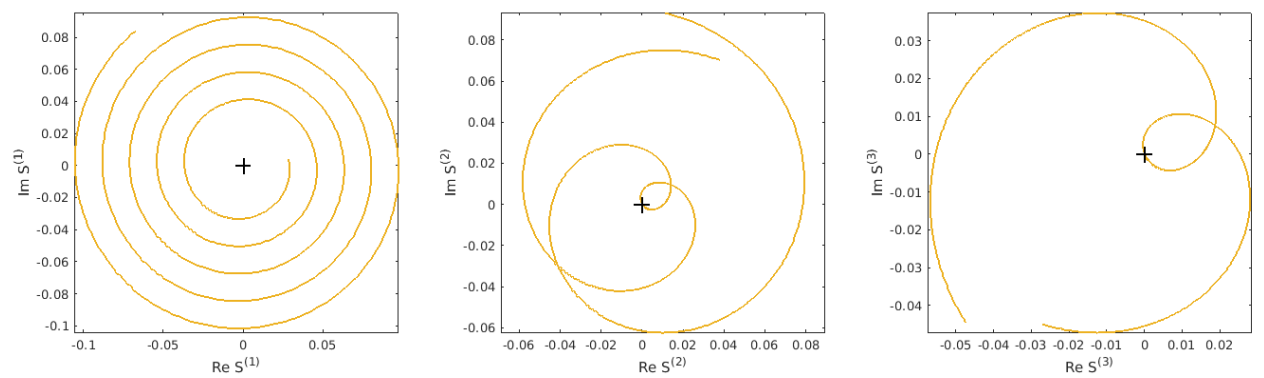

(a) First order fitting: $P=Q=1$, sum of squared residuals $=0.081$.
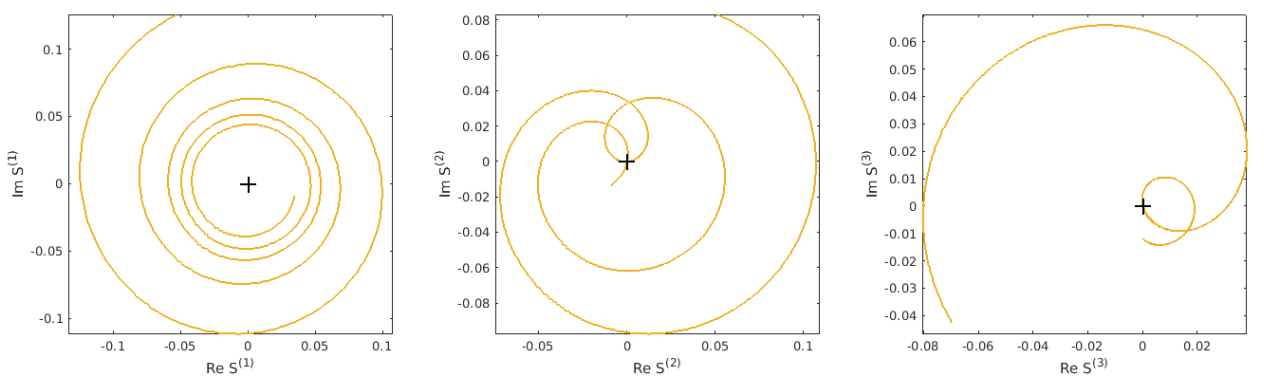

(b) Higher order fitting: $P=Q=3$, sum of squared residuals $=2.37 \times 10^{-6}$.

Figure 8: S-parameter components from (a) first order and (b) higher order fitting without regularization, same instantiation starting from the same initialization. Even though the higher order fitting significantly lowers the residuals, its components have much more complicated shapes, suggesting overfitting. We use regularization as described in Section 4.3 to guard against such overfitting.

\section{RESIDUAL DISTRIBUTION AND POST-PROCESSING}

Functioning just like the feature parameters of a resonator response, the polynomial coefficients $\mathbf{a}, \mathbf{p}$ can be directly used with statistical modeling as a substitute for the full S-parameter response, but only when the fitting residual is negligible. In practice, since the actual component responses are not strictly simple polynomials, and there may exist many other minor components that are hard to separate, the fitting may not be able to eliminate all of the residual. Modeling the residual is even harder than the original S-parameters. However, if we treat the residual as additional noise or variation and apply it to the decomposed component, it can be linearized and estimated by the statistical tools. In other words, instead of using the decomposition

$$
S(f)=\sum_{i=1}^{n} \hat{S}\left(f ; a^{(i)}, p^{(i)}\right)+r(f)
$$

and modeling $\mathbf{a}, \mathbf{p}, r(f)$ jointly, we can distribute the residual $r(f)$ to each component $S^{(i)}$, so that

$$
S(f)=\sum_{i=1}^{n} S^{(i)}(f)=\sum_{i=1}^{n}\left(\hat{S}\left(f ; a^{(i)}, p^{(i)}\right)+r^{(i)}(f)\right)
$$

with $\sum_{i=1}^{n} r^{(i)}(f)=r(f)$, and then perform statistical analysis on $S^{(i)}$.

Dividing the residual $r(f)$ into the parts $r^{(i)}(f)$ is a problem with many requirements to satisfy. Firstly, $r^{(i)}(f)$ should be small compared to $\hat{S}^{(i)}(f)$, so that the assumption of linearization still holds for the statistical 
analysis. Secondly, for the component $\hat{S}^{(i)}(f)$ with trajectory close to the origin at some certain frequency $f_{o}$, $r^{(i)}\left(f_{o}\right)$ should be almost zero, as any tiny change close to the origin can introduce a huge spike in group delay response. Lastly, after the distribution, $S^{(i)}(f)$ should be at least first order differentiable. According to these requirements, we propose a frequency-wise, amplitude-proportional distribution scheme:

$$
r^{(i)}(f)=\frac{\left|\hat{A}\left(f ; a^{(i)}\right)\right|^{2}}{\sum_{i^{\prime}=1}^{n}\left|\hat{A}\left(f ; a^{\left(i^{\prime}\right)}\right)\right|^{2}} r(f) .
$$

We can formulate each component $S^{(i)}$ in the amplitude-phase representation and combine them together to the decomposed representation (3) for $S$. However, since we introduce the "noisy" residual to each simple Sparameter, it causes ripples and spikes in the group delay response that may make the decomposition suboptimal. Shifting each component by a small number, as suggested at the beginning of Section 3, can be adopted here since it works for simple S-parameters; i.e., find a complex number $c_{i}$ for each component $S^{(i)}$ such that

$$
\tilde{S}^{(i)}(f)=S^{(i)}(f)-c_{i}
$$

has the least amount of ripples in its group delay response.

Letting $\tilde{\phi}_{j}=\tilde{\phi}\left(f_{j}\right)=\arg \left(\tilde{S}\left(f_{j}\right)\right)$, we estimate the group delay of $\tilde{S}(f)$ from finite difference method:

$$
\tilde{g}_{j+1 / 2}=\frac{1}{2 \pi} \frac{\mathrm{d} \tilde{\phi}}{\mathrm{d} f} \approx \frac{\tilde{\phi}_{j+1}-\tilde{\phi}_{j}}{2 \pi \Delta f} \propto \tilde{\phi}_{j+1}-\tilde{\phi}_{j}
$$

It should be noted that if $c_{i}$ is arbitrarily large, then $\tilde{S}(f)$ has an almost constant phase response, and thus has small amount of change in group delay. To eliminate this case in the optimization, we introduce a regularization term based on the correlation between $\tilde{\phi}(f)$ and $f$, as the desired $\tilde{S}(f)$ has nonzero and almost constant group delay, and thus $\tilde{\phi}(f)$ should have strongly linear dependence on $f$ (see Figure 5b and Figure $6 \mathrm{~b}$ ). We then formulate the optimization problem as minimizing the maximal difference in group delay (19):

$$
\begin{array}{rl}
c_{i}^{*}=\min _{c_{i}} & G-g+\lambda\left(1-\rho_{\tilde{\phi}, f}^{2}\right) \\
\text { s.t. } & \tilde{\phi}_{j}=\arg \left(S^{(i)}\left(f_{j}\right)-c_{i}\right) \\
& G \geq \tilde{\phi}_{j+1}-\tilde{\phi}_{j} \geq g .
\end{array}
$$

For the Type II simple S-parameters, we also need to make sure that $\tilde{S}(f)$ is still a Type II simple S-parameter, i.e., it also crosses the origin exactly. Therefore, in this case, $c_{i}$ must be on the trajectory of $S^{(i)}(f)$, thus we can parameterize it as $c_{i}=S^{(i)}\left(f_{o}\right)$ and optimize for $f_{o}$. The formulation is very similar to (20), but we need to amend the $\pi$ jump at $f_{o}$ when calculating $\tilde{\phi}$ as mentioned in Section 3. In addition, since the phase is sensitive to small changes near the origin, the finite difference estimation (19) becomes noisy around $f_{o}$. Thus the frequency segments very close to $f_{o}$ should be discarded and replaced by the interpolation from other data in the group delay calculation.

After shifting, the new decomposition becomes

$$
S(f)=\sum_{i=1}^{n}\left(\tilde{S}^{(i)}(f)+c_{i}\right)=\sum_{i=1}^{n} \tilde{S}^{(i)}(f)+c
$$

with an additional complex shifting $c=\sum_{i=1}^{n} c_{i}$. Since the shifting can be treated as an $(n+1)$-th component with constant amplitude and phase response, it can follow the same procedure in the statistical analysis as in the prior decomposed representation. 


\section{TESTING WITH PRINCIPAL COMPONENT ANALYSIS}

We use an ensemble of 50 instantiations of Y-splitters with LER parameters $A=10 \mathrm{~nm}, L_{c}=40 \mathrm{~nm}$ (Figure 3 ) as an example and perform the above decomposition on all of the ensemble instantiations. We then compare the PCA results based on the conventional amplitude-phase representation versus that using the proposed decomposed representation. PCA is performed by applying singular value decomposition (SVD) ${ }^{16}$ upon all the representation components at all the frequency points jointly. Due to the highly nonlinear behavior in group delay (Figure 3b), SVD upon the traditional amplitude-phase representation does not exhibit any apparent cutoff threshold in the singular value distribution (purple dashed line in Figure 9a); in contrast, SVD upon our decomposed representation has a clear cutoff threshold at the 10-th component.

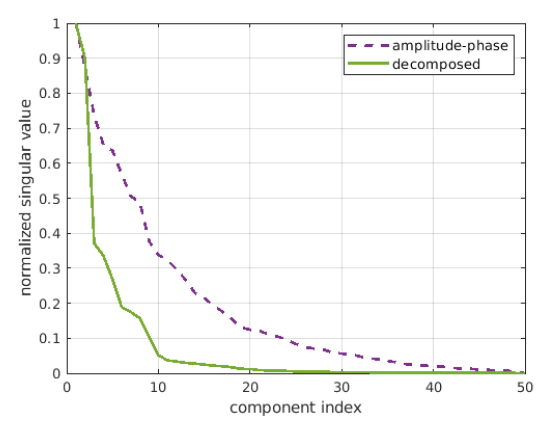

(a)

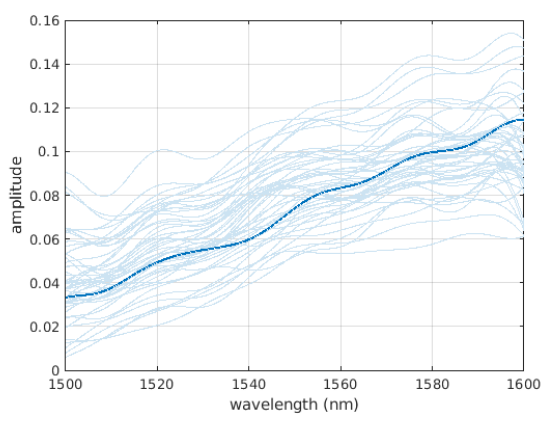

(b)

Figure 9: (a) Singular values from SVD using the conventional amplitude-phase representation (purple dashed) and the proposed decomposed representation (green solid), component ordered by singular value, and normalized by the maximum value for comparison. (b) The amplitude response $A^{(1)}$ in the decomposed representation (3), generated from the first 10 components in the SVD.

We next generate samples from the first 10 components in the SVD for both representations. As shown in Figure 10, the generated S-parameters based on the conventional amplitude-phase representation may have nonphysical jagged group delay response (Figure 10b) and a discontinuous trajectory in the complex plane (Figure 10c). In contrast, generated S-parameters based on our decomposed representation always have smooth, physically appropriate response (Figure 11). The distribution of the 50 instantiations in Figure 11a and Figure 11b is also close to that of the original simulated ensemble (Figure 3a and Figure 3b), though noting that some instantiations in Figure 11a have an unusually large amplitude near $1500 \mathrm{~nm}$.

It is also worth noting that in the generated samples, the amplitude response of the S-parameter component, $A^{(i)}$ in the decomposed representation (3), remains the small ripples behavior (Figure 9b) that is the result of residual distribution. This is the evidence that the fitting residual is identified and included in the first 10 components in SVD, and thus residual distribution is necessary. 


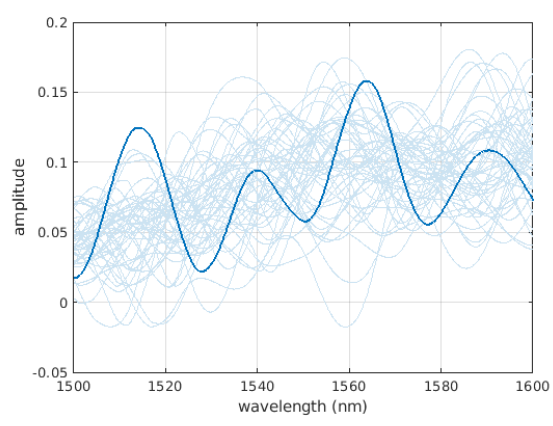

(a) amplitude

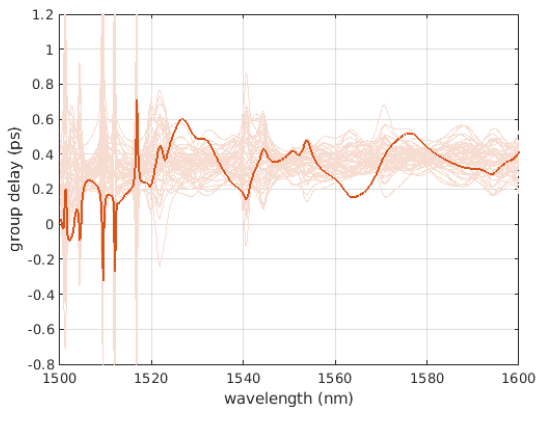

(b) group delay

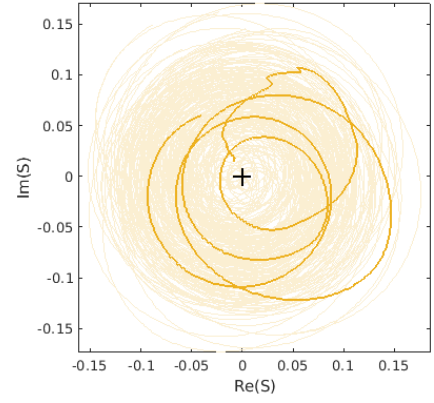

(c) trajectory

Figure 10: Frequency response and S-parameter trajectory of the PCA generated Y-splitter arm back reflection with LER of $A=10 \mathrm{~nm}, L_{c}=40 \mathrm{~nm}$ based on the data from Figure 3, using conventional amplitude-phase representation, 50 instantiations, with one instantiation highlighted showing typical group delay artifacts.

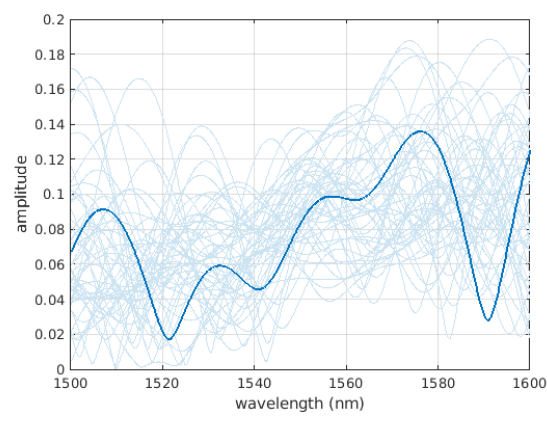

(a) amplitude

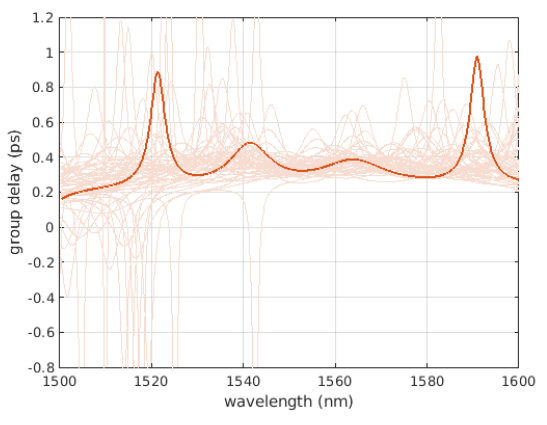

(b) group delay

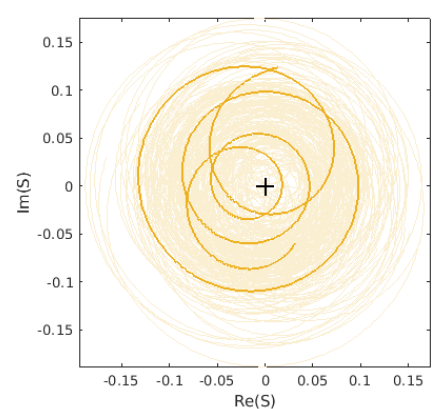

(c) trajectory

Figure 11: Frequency response and S-parameter trajectory of the PCA generated Y-splitter arm back reflection with LER of $A=10 \mathrm{~nm}, L_{c}=40 \mathrm{~nm}$ based on the data from Figure 3, using our proposed decomposed representation, 50 instantiations, with one instantiation highlighted. Artifacts in amplitude and group delay are eliminated.

\section{CONCLUSIONS}

We present a novel decomposed representation for photonic device S-parameters that explains complicated Sparameters by decomposition into several simple components, thereby overcoming high-nonlinearity barriers in the statistical analysis of variation across ensembles of devices. We present methods to determine the decomposed representation through regularized fitting, and show its improvement in conjunction with PCA for statistical compact model description of device variation.

A case study is presented applying PCA to S-parameters based on both the traditional amplitude-phase representation and our proposed decomposed representation of Y-splitters with applied line edge roughness. Our proposed representation successfully truncates at low order (small number of PCA components), and has better performance in capturing the variations and generating physically realistic samples. The same method can be applied to any photonic device component S-parameter that is the result of interference of several components, which enables applying much simpler statistical analysis (e.g., using PCA) instead of complicated non-linear statistical analysis.

Future works could seek to eradicate the causality dilemma between ensemble parameters statistic and regularized fitting mentioned in Section 4.2, and extend the application to cases with multiple ensembles of different variation parameters, e.g., LER with different amplitude and correlation lengths. 


\section{REFERENCES}

[1] Bowers, J. E., Komljenovic, T., Davenport, M., Hulme, J., Liu, A. Y., Santis, C. T., Spott, A., Srinivasan, S., Stanton, E. J., and Zhang, C., "Recent advances in silicon photonic integrated circuits," in [Next-Generation Optical Communication: Components, Sub-Systems, and Systems V], 9774, 977402, International Society for Optics and Photonics (2016).

[2] Sun, C., Wade, M. T., Lee, Y., Orcutt, J. S., Alloatti, L., Georgas, M. S., Waterman, A. S., Shainline, J. M., Avizienis, R. R., Lin, S., et al., "Single-chip microprocessor that communicates directly using light," Nature 528(7583), 534 (2015).

[3] Martinez, G., Methods for Compact Modeling of Process Variations in Silicon Photonics Devices, Master's thesis, Massachusetts Institute of Technology (2018).

[4] El-Henawy, S. I., Zhang, Z., Miller, R., and Boning, D. S., "Photonic device sensitivity analysis methods: towards process variation-aware silicon photonics design," in [Optical Modeling and System Alignment], 11103, 111030P, International Society for Optics and Photonics (2019).

[5] Zhang, Z., El-Henawy, S. I., Sadun, A., Miller, R., Daniel, L., White, J., and Boning, D., "Adjoint-Based sensitivity analysis for silicon photonic variations," in [IEEE MTT-S International Conference on Numerical Electromagnetic and Multiphysics Modeling and Optimization (NEMO 2019)], (May 2019).

[6] Melati, D., Lovati, E., and Melloni, A., "Statistical process design kits: Analysis of fabrication tolerances in integrated photonic circuits," Integrated Photonics Research, Silicon and Nanophotonics, IPRSN 20151 , $371 \mathrm{p}(2015)$.

[7] El-Henawy, S. I., Lang, C., and Boning, D. S., "Yield prediction for coupled-resonator optical waveguides using variation-aware compact models," in [Frontiers in Optics], FTu1C-3, Optical Society of America (2019).

[8] Chrostowski, L. and Hochberg, M., [Silicon Photonics Design: From Devices to Systems], Cambridge University Press (2015).

[9] El-Henawy, S. I., Miller, R., and Boning, D. S., "Effects of a random process variation on the transfer characteristics of a fundamental photonic integrated circuit component," in [Proc. of SPIE], 10743, 107430O-1 (2018).

[10] Zhang, Y., Yang, S., Lim, A. E.-J., Lo, G.-Q., Galland, C., Baehr-Jones, T., and Hochberg, M., "A compact and low loss Y-junction for submicron silicon waveguide.," Optics Express 21(1), 1310-6 (2013).

[11] Wold, S., Esbensen, K., and Geladi, P., "Principal component analysis," Chemometrics and intelligent laboratory systems 2(1-3), 37-52 (1987).

[12] "SEMulator3D 7.1, Coventor Inc.."

[13] Available:. https://www.lumerical.com/products/fdtd-solutions.

[14] Yariv, A., "Universal relations for coupling of optical power between microresonators and dielectric waveguides," Electronics letters 36(4), 321-322 (2000).

[15] Tibshirani, R., "Regression shrinkage and selection via the lasso," Journal of the Royal Statistical Society: Series B (Methodological) 58(1), 267-288 (1996).

[16] Golub, G. H. and Reinsch, C., "Singular value decomposition and least squares solutions," in [Linear Algebra], 134-151, Springer (1971). 